\title{
Cerebrospinal fluid velocity changes of idiopathic scoliosis: a preliminary study on 3-T PC-MRI and 3D-SPACE-VFAM data
}

\author{
O. Algin ${ }^{1,2,3(1)} \cdot$ U. $\operatorname{Koc}^{4} \cdot$ N. Yalcin ${ }^{5}$
}

Received: 6 December 2020 / Accepted: 21 August 2021 / Published online: 6 October 2021

(c) The Author(s), under exclusive licence to Springer-Verlag GmbH Germany, part of Springer Nature 2021

\begin{abstract}
Objectives To the best of our knowledge, there is no study on 3-Tesla (3-T) phase-contrast MRI (PC-MRI) and three-dimensional sampling perfection with application-optimized contrasts using different flip-angle evolutions (3D-SPACE-VFAM) in the evaluation of idiopathic scoliosis. This study aimed to investigate CSF abnormalities in the scoliotic spine using 3-T PCMRI and 3D-SPACE-VFAM techniques.

Methods Thirty-four patients and 14 controls were examined with spinal PC-MRI and T2-weighted 3D-SPACE-VFAM techniques. Inter- and intra-reader agreements of flow-void phenomenon on 3D-SPACE-VFAM images, and velocity values on PC-MRI data were also evaluated.

Results There are statistically significant differences between scoliosis and control groups based on the highest and mean peak velocity values on PC-MRI images ( $p=0.005$ and $p=0.023$, respectively). The main thoracic (MT) group's highest peak CSF velocity values were higher than the control group $(p=0.022)$. There is a significant difference between the patient and control groups regarding flow-void phenomenon scores on 3D-SPACE-VFAM images $(p=0.036)$. Inter- and intra-reader agreement values related to PC-MRI velocity measurements were perfect for all PC-MRI readings. Inter- and intra-reader agreement values of the flow-void phenomenon scores were moderate.

Conclusions Our study has led us to conclude that idiopathic scoliosis is associated with CSF flow disturbances in parallel with the literature. MRI can demonstrate these abnormalities in a non-invasive and radiation-free way.
\end{abstract}

Keywords PC-MRI $\cdot$ CSF $\cdot$ Idiopathic scoliosis $\cdot$ Magnetic resonance imaging $\cdot 3$-Tesla $\cdot 3 \mathrm{D}$-SPACE

Advances in knowledge The roles of 3-tesla 3D-SPACE-VFAM and PC-MRI examinations in the evaluation of idiopathic scoliosis are unknown. Our results showed that hyperdynamic CSF flow is present in patients with idiopathic scoliosis.

O. Algin

droktayalgin@gmail.com

1 Department of Radiology, City Hospital, Bilkent, Ankara, Turkey

2 Department of Radiology, Yildirim Beyazit University, Ankara, Turkey

3 National MR Research Center, Bilkent University, Ankara, Turkey

4 City Hospital, Department of Radiology, Bilkent, Ankara; and General Directorate of Health Information Systems, Ministry of Health, Ankara, Turkey

5 Department of Orthopedics and Traumatology, Karabük University, Karabük, Turkey

\section{Introduction}

Idiopathic scoliosis (IS) affects around $4 \%$ of children globally [1-3]. It is observed ten times higher in girls than in boys (especially in patients with Cobb angles $>30^{\circ}$ ) [1-3]. Substantial research efforts have been directed to investigate possible mechanisms, none of which explain all the different facets of this complex disease. There is strong evidence of a familial/genetic background, but genetic studies have failed to identify any single genetic locus or even a single chromosome contributing to IS [2-4].

Several theories and factors have been proposed to explain the multifactorial pathogenesis of IS. These are connective tissue disorders, skeletal muscle or contractile tissue disorders, developmental imbalance, the involvement of the vestibular and proprioceptive systems, hormonal factors, uncoupled neuro-osseous growth, biomechanical issues, and dissociation between the timing of the skeletal and central nervous system maturation [1-5]. 
In experimental studies, cerebrospinal fluid (CSF) flow changes and abnormal cilia motility were found and related to spinal deformity and scoliosis $[6,7]$. Also, protein tyrosine kinase 7 (ptk7) gene mutation causes scoliosis in the zebrafish model [4]. It was found that the ptk7 gene, pkd211 channels, and CSF-contacting neurons are related to the ciliary function and CSF flow [7]. These studies have provided a paradigm change in the etiopathogenesis of scoliosis and lead to new research areas. However, we still do not know what causes scoliosis, and abnormal CSF flow patterns in humans could cause or affect IS.

Isotropic 3D-SPACE with variant flip-angle mode (3D-SPACE-VFAM) is a recently developed T2-weighted (W) turbo spin-echo technique [8]. The 3D-SPACE-VFAM and phase-contrast cine MRI (PC-MRI) are non-invasive and useful MRI techniques for evaluating CSF flow [8]. Therefore, we focused on evaluating CSF flow changes in patients with IS by 3-T PC-MRI and 3D-SPACE-VFAM techniques. According to our knowledge, detailed information about the roles of 3-T 3D-SPACE-VFAM and PC-MRI examinations in the evaluation of IS is unknown. Also, reliabilities of thoracal or lumbar 3D-SPACE-VFAM and PC-MRI exams are currently missing.

The aim of this preliminary study was twofold: first, to investigate the CSF flow changes in the scoliotic spine and compare them with controls with a non-invasive and radiation-free modality, MRI. Second, determine the intra-reader and inter-reader agreement values of spinal 3D-SPACEVFAM and PC-MRI exams.

\section{Materials and methods}

\section{Study population}

Local ethics committee approval was obtained for this prospective study. Informed consent was obtained from all cases before the imaging sessions. No invasive procedure was applied to any patient for this study.

\section{Inclusion criteria}

- Patients with IS,

- Patients have 3-Tesla (3-T) spinal PC-MRI and T2W 3D-SPACE sequences.

\section{Exclusion criteria}

- Absence of IS,

- Patients with congenital, neurogenic, or degenerative scoliosis,

- Insufficient MR image quality to evaluate spinal CSF flow due to artifacts,
- Patients with MRI contraindications (e.g., claustrophobia, pacemaker),

- Pregnant cases,

- Patients with a history of spinal, pelvic, or cranial surgery.

The final study population included 34 patients who were referred to our center in 5 years. Besides, 14 controls were scanned with the same MRI protocol in this period. All the patients with IS were referred for clinically indicated MRI, and the controls were clinically indicated MRI for some other reasons.

\section{Imaging protocols and image analyses}

All MR examinations were performed on a 3-T MR scanner (Trio, Siemens Healthcare, Erlangen, Germany) using spine and body matrix coils. After acquiring localizer images, isotropic high-resolution T2W 3D-SPACE-VFAM, T1W 3D-SPACE, and PC-MRI data were obtained. The acquisition parameters of the MRI exams are given in Table 1. All patients and controls were scanned in a supine position with no pre-imaging preparation.

3D-SPACE-VFAM images were acquired to include all thoracolumbar spines. Single-slab cardiac gated cine through-plane PC-MRI data were obtained from lower thoracic level (for patients with the only lumbar curve) and scoliotic apex (for patients with a thoracic curve). Reader 1 (O.A. with 12 years of experience in 3D-SPACE and PCMRI) rotated and evaluated the 3D-SPACE-VFAM images

Table 1 Three-Tesla MRI protocol of the study

\begin{tabular}{llll}
\hline Sequences/parameters & T1-TSE & 3D-SPACE & PC-MRI \\
\hline TR/TE (ms) & $650 / 9.8$ & $3000 / 279$ & $29.25 / 7.03$ \\
Slice thickness (mm) & 3 & 1.5 & 3 \\
FOV* $(\mathrm{mm})^{\text {Acquisition time (minute) }}$ & 1.57 & 8 & $190 \times 190$ \\
NEX & 2 & 1 & 3.48 \\
Number of slices & 15 & 44 & 1 \\
Flip angle $\left({ }^{\circ}\right)$ & 150 & 100 & 1 \\
Imaging plane & Sagittal & Sagittal & Axial \\
PAT factor & 2 & 3 & - \\
Voxel size (mm) & $0.7 \times 0.5 \times 3$ & $1 \times 1 \times 1.5$ & $0.7 \times 0.7 \times 3$ \\
FA mode & - & T2 variant & - \\
Phase encoding direction & HF & HF & AP \\
PAT mode & Grappa & Grappa & - \\
Velocity encoding value & - & - & $20 \mathrm{~cm} / \mathrm{s}$ \\
Excitation & - & Slab-selective & - \\
\hline
\end{tabular}

$3 D$-SPACE three-dimensional sampling perfection with applicationoptimized contrasts using different flip-angle evolutions, T1-TSE T1W turbo spin-echo, NEX number of excitations, FOV field of view, $P A T$ parallel acquisition technique

* The FOV value may vary depending on the size of the patient 
using the multiplanar reformat (MPR) technique. The flowvoid phenomenon observed at the subarachnoid space was investigated on 3D-SPACE-VFAM images. This phenomenon was scored visually, as described below.

Grade 1: none (there is no hypointense flow through the subarachnoid space on 3D-SPACE-VFAM data).

Grade 2: minimal (the flow-void phenomenon is barely visible).

Grade 3: moderate (a visible flow-void phenomenon).

Grade 4: prominent (prominent and multiple hypointense CSF flow-void signals along with the entire thoracolumbar subarachnoid space).

PC-MRI data were processed in the ARGUS software (Siemens Healthcare, Germany), and flow/velocity parameters were determined by reader 1 . Twenty-six rephased, magnitude, and phase images were obtained from each PCMRI acquisition. Although PC-MRI sequence acquisition time varied according to the subjects' heart rates, each PCMRI sequence's duration was approximately $4 \mathrm{~min}$. A saturation band was placed at the aortic level to prevent aliasing artifacts (Fig. 1).

Three regions of interest (ROIs) were placed in the entire spinal canal, including the whole subarachnoid space on axial plane PC-MRI phase images. Special attention was paid to exclude intradural/perimedullary vessels and bones. Another circular ROI was also drawn on the vertebra corpus (for determination of spine mobility) and muscle (as a reference ROI). Care was taken to ensure that the areas of the ROIs were as equal as possible. The ARGUS software calculated the velocity in the regions of interest in all time frames.

Anterior-posterior and lateral spinal X-ray images of the patients were retrospectively retrieved from our picture archival and communications system (PACS). These X-ray images are standardized and evaluated by an experienced orthopedist (N.Y.) and radiologist (O.A.). Cobb angles were calculated, and the curves were classified according to the Lenke classification system $[1,5]$.
The flow-void phenomenon scores and PC-MRI measurements were re-evaluated separately by reader 1 to demonstrate intra-reader agreement values. Also, these parameters were assessed by reader 2 (5-year experience in radiology) to determine inter-reader agreements. Second measurements of the more experienced reader were considered the goldstandard test for PC-MRI measurements and 3D-SPACEVFAM scorings.

\section{Statistical analyses}

Comparison of categoric and nominal data was evaluated by the chi-square or Fisher's exact test, while numeric data between groups by Mann Whitney $U$ and Kruskal Wallis test. Agreements between readers were evaluated by the Intraclass Correlation coefficient (ICC) and Cohen's Kappa (к) statistics. Spearman Correlation test was applied for correlation analysis between nominal, categoric, and numeric data. SPPS for Windows software package (version 21.0, SPSS Inc.) was used for statistical analysis. $P$-Value $<0.05$ was accepted as statistically significant.

\section{Results}

It was found that there was no significant difference between the groups in terms of gender and age (Table 2). Cobb angles of the patients are given in Table 2. PC-MRI images revealed a sinusoidal black and white flow pattern (representing up and down movement) in the subarachnoid space of the spine of all the cases (Fig. 2). Curve types, localizations, and lumbar modifiers of the patients are given in Tables 2 and 3 .

There are statistically significant differences between patient and control groups based on the highest and mean peak velocity values on PC-MRI images ( $p=0.005$ and $p=0.023$, respectively) (Table 4 ). When the patients were further analyzed in terms of Lenke types, the highest peak CSF velocity values of the main thoracic (MT) group were higher than the control group ( $p=0.022)$ (Fig. 3 and 4$)$. There was no significant difference between the groups in terms
Fig. 1 A saturation band was placed just anterior to the vertebral column on coronal (A) axial (B) sagittal (C) plane images for PC-MRI acquisitions

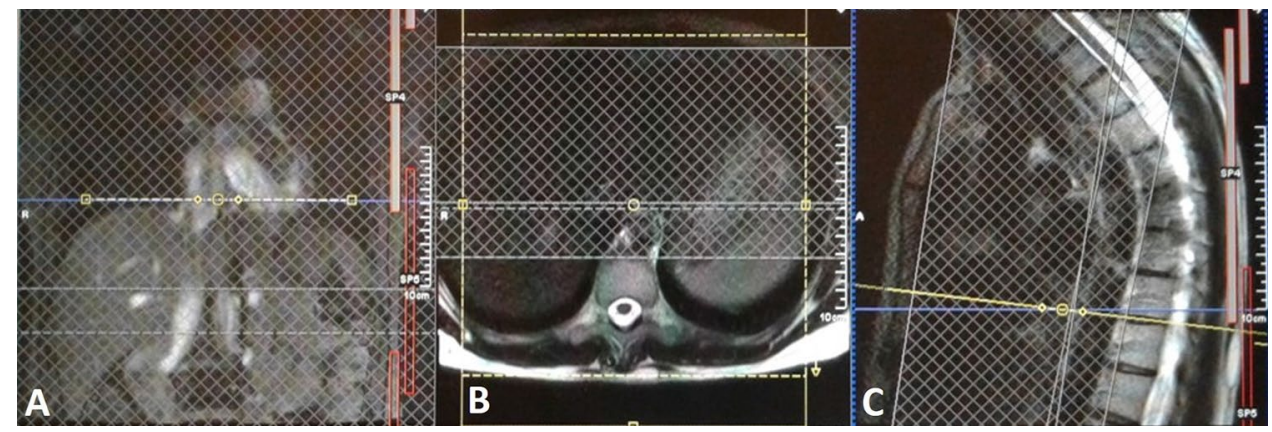


Table 2 Curve types/localizations and demographic values of the patients

\begin{tabular}{lll}
\hline Sex & CONTROLS $(\mathrm{n}=14)$ & PATIENTS $(\mathrm{n}=34)$ \\
\hline Male & $4(71.4)$ & $10(70.6)$ \\
Female & $10(28.6)$ & $24(29.4)$ \\
Age & $19.93 \pm 4.09$ & $17.7 \pm 7.9$ \\
& $(20 ; 15-26)$ & $(16 ; 10-48)$ \\
Lumbar Cobb Angle & - & $35.12 \pm 18.16$ \\
& & $(33.7 ; 13.7-81.9)$ \\
Thoracic Cobb Angle & - & $27.27 \pm 14.47$ \\
& & $(23.5 ; 10.0-65.8)$ \\
Curve Type & CONTROLS $(\mathrm{n}=14)$ & PATIENTS $(\mathrm{n}=34)$ \\
Main Thoracic (MT1) & - & $18(52.9)$ \\
Thoraco-lumbar 5 (TL5) & - & $10(29.4)$ \\
Lumbar 5 (L5) & - & $4(11.8)$ \\
Double Thoracic (DT2) & - & $1(2.9)$ \\
Lumbar 6 (L6) & - & $1(2.9)$ \\
Lumbar Spine Modifier & CONTROLS $(\mathrm{n}=14)$ & PATIENTS $(\mathrm{n}=34)$ \\
A & - & $16(47.06)$ \\
B & - & $5(14.71)$ \\
C & - & $13(38.24)$ \\
Sagittal Thoracic & CONTROLS $(\mathrm{n}=14)$ & PATIENTS $(\mathrm{n}=34)$ \\
Modifier & & $2(5.88)$ \\
- & - & $2(5.88)$ \\
+ & - & $30(88.24)$ \\
N & &
\end{tabular}

Age and Cobb angle parameters are shown as mean \pm standard deviation (median; minimum-maximum), while other nominal and categorical data are numbers (n) with the percentage in parentheses

of the other CSF velocity parameters. CSF velocity values of the patient and control groups are given in Table 4.

There is a strong positive correlation between thoracic and lumbar Cobb angles in the patient group $(r=0.700$, $p=0.036$ ). Also, there was an inverse relationship between thoracic angles and the highest maximum spine movement (mobility) values $(r=-0.441, p=0.035)$. Spine mobility values on PC-MRI images of the groups are given in Table 4 . We did not find any statistical differences between patient and control groups regarding spine mobility measurements on PC-MRI images.

The flow-void phenomenon scores on 3D-SPACEVFAM images differed between the two groups, which was statistically significant ( $p=0.036$, Fig. 3 ). The flow-void phenomenon scores of the groups are given in Table 5.

Inter- and intra-reader agreement values related to PCMRI velocity measurements were perfect for all PC-MRI readings (Fig. 4). Besides, inter-and intra-reader agreement values of the flow-void phenomenon scores are moderate (Table 6).

\section{Discussion}

IS is the most common (84-89\%) form of scoliosis and is frequently seen in young people and called adolescent IS [9]. The etiology or mechanism of scoliosis is not fully understood [2]. The most common etiopathogenesis of IS includes maturation disorders of the central and peripheral nervous system, connective tissue disorders in elastic and collagen fibers, muscle and bone diseases, platelet disorders, melatonin, hormone imbalances, genetic abnormalities, and leptin deficiency (essential in central nervous system development) [9-11]. Also, single nucleotide polymorphisms or any mutation related to ciliary functions and growth-regulating proteins may be related to IS development and progression [11-13].

It is supposed that there is a link between CSF flow disturbances and IS [4, 13]. Mutations of ptk7 lead to a lack of ciliary activity and neuroinflammatory responses at the onset of spinal curve formation in zebrafish studies [2]. Experimental studies in rabbits showed that CSF flow disorders might be related to scoliosis. Takano et al. found that the highest CSF flow was faster at the apical vertebra in the adolescent IS patients $(8.88 \pm 3.81 \mathrm{~cm} / \mathrm{s})$, compared with at the apical vertebra level of $\mathrm{T} 8$ in controls $(6.53 \pm 2.47 \mathrm{~cm} / \mathrm{s})$ [14]. Also, they reported that there was a significant correlation between the Cobb angle and the peak CSF velocity at the apical vertebra $(r=0.77$, $p<0.01)$ in their preliminary 1.5-T MR unit results [14]. Our results showed that CSF peak velocity values in patients with IS differ compared to the controls in concordance with these studies.

Scaramuzzo et al. reported that neuro-axial abnormality (e.g., hydromyelia, syringomyelia, or Chiari I) incidence is $26 \%$ in patients with IS [15]. $15 \%$ of patients with IS and normal neurological examination will demonstrate a neuro-axial abnormality on spinal MRI [16]. A preliminary study also reported that 3D MRI sequences are more accurate than computed tomography (CT) in preoperative measurements of patients with scoliosis [5]. 3D-SPACE images may be a valuable alternative to CT for the evaluation of patients with IS. As observed in our study, the flow-void phenomenon indicates turbulent or complex flow due to scoliotic CSF disturbances in most IS cases.

IS occurs predominantly in adolescents [10, 17]. MRI is a radiation-free technique. This is an important advantage for repeated examinations [17]. Highresolution multiplanar or oblique reformatted images of 3D-SPACE-VFAM are beneficial and usually sufficient for morphological assessment of scoliosis in pre-and postoperative periods. Dissemination of this technique and pooling data across multiple centers may lead to a more efficient and reliable knowledge about diagnosis, effective 


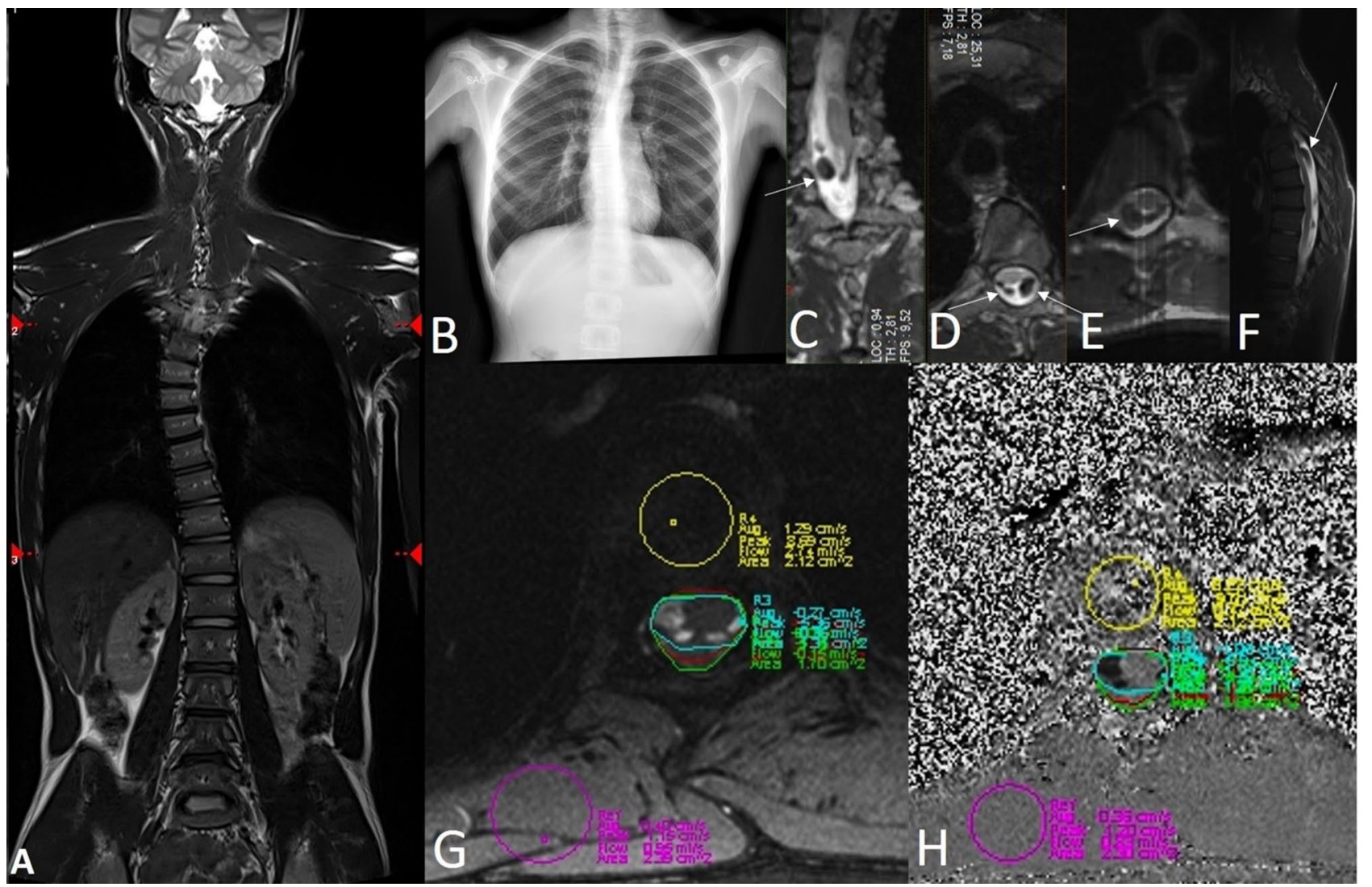

Fig. 2 3D-SPACE-VFAM (A) and PA X-ray (B) images of a patient with idiopathic scoliosis. There are multiple flow-void signals on reformatted 3D-SPACE-VFAM images consistent with the grade 3 flow-void phenomenon (arrows in $\mathbf{C}-\mathbf{F}$ ). There are five ROIs on PCMRI images for demonstrations of velocity values $(\mathbf{G}, \mathbf{H})$. Yellow

treatment (observation, bracing, or elective surgery), and non-invasive follow-up of the patients with IS. Evaluation of IS cases within the post-operative period with CT or MRI is often tricky because of artifacts related to the surgical instruments. 3D-SPACE imaging is more resistant to artifacts due to its turbo spin-echo nature. According to our experiences, this technique with multiple viewing
ROI was placed for spine mobility. Pink ROI was drawn as a reference ROI. Other ROIs were used for CSF measurements. The highest peak CSF velocity is $10.04 \mathrm{~cm} / \mathrm{s}$ and average CSF velocity is $0.3 \mathrm{~cm} / \mathrm{s}$ on these measurements. The highest max. spine movement was $9.85 \mathrm{~cm} / \mathrm{s}$

angles is useful for demonstrating changes related to scoliosis or surgical instruments.

Our study demonstrates the feasibility of applying PCMRI and 3D-SPACE-VFAM to evaluate patients with IS with good to perfect agreement or reliability values. We believe that, as the experience increases, the reliability of these techniques will further increase.
Table 3 Curve types of the patients according to Lenke classification $(\mathrm{N} / \mathrm{A}=$ not applicable)

\begin{tabular}{llcll}
\hline Lenke Classification & $\begin{array}{l}\text { Number of } \\
\text { cases }\end{array}$ & & $\%$ of cases & \multicolumn{2}{l}{$\begin{array}{l}\text { Mean (min-max) degree of curve } \\
\text { magnitude }\end{array}$} \\
\cline { 3 - 5 } & & & Thoracic & Lumbar \\
\hline Type 1 (Main thoracic) & 18 & 52.94 & $29.51(10-65.8)$ & N/A \\
Type 2 (Double thoracic) & 1 & 2.94 & 46.6 and 48.4 & N/A \\
Type 3 (Double major) & - & - & - & - \\
Type 4 (Triple major) & - & - & - & - \\
Type 5 (Thoracolumbar/Lumbar) & 14 & 41.18 & $20.37(13.5-25.8)$ & $34.14(13.7-81.9)$ \\
Type 6 (Thoracolumbar/Lumbar- & 1 & 2.94 & 19.8 & 21.8 \\
Main Thoracic) & & & & \\
\hline
\end{tabular}


Table 4 PC-MRI parameters and flow-void phenomenon scores of the groups. Note-data are mean \pm standard deviation with median; minimum-maximum in parentheses (except for flow-void scores)

\begin{tabular}{|c|c|c|c|}
\hline & Controls $(n=14)$ & Patients $(n=34)$ & $p$ Value \\
\hline \multicolumn{4}{|l|}{ Parameters } \\
\hline Highest peak & $7.38 \pm 2.27(7.4 ; 3.93-10.94)$ & $10.20 \pm 3.51(9.37 ; 5.23-23.84)$ & 0.005 \\
\hline Mean peak & $5.81 \pm 1.42(5.96 ; 3.52-7.83)$ & $7.53 \pm 2.53(6.95 ; 4.81-17.39)$ & 0.023 \\
\hline Average & $0.77 \pm 0.35(0.72 ; 0.27-1.50)$ & $0.58 \pm 0.31(0.58 ; 0.09-1.20)$ & 0.12 \\
\hline Highest max. spine movement & $11.89 \pm 4.17(12.9 ; 3.84-17.0)$ & $10.80 \pm 3.85(10.56 ; 3.62-23.65)$ & 0.2 \\
\hline Maximum spine movement & $9.82 \pm 3.18(10.1 ; 3.84-14.0)$ & $8.63 \pm 3.43(8.85 ; 0.26-17.57)$ & 0.14 \\
\hline Average spine movement & $0.51 \pm 0.28(0.44 ; 0.17-0.96)$ & $0.58 \pm 0.38(0.53 ; 0.11-1.90)$ & 0.812 \\
\hline \multicolumn{4}{|l|}{ Flow-void scores } \\
\hline 1 & 2 & 2 & 0.036 \\
\hline 2 & 3 & 7 & \\
\hline 3 & 1 & 17 & \\
\hline 4 & 0 & 8 & \\
\hline
\end{tabular}

PC-MRI method has several disadvantages, including $2 \mathrm{D}$ (single-slab) acquisition capability, experience requirement, and the same contraindications of MRI [8]. Similarly, 3D-SPACE-VFAM is slower than CT. It also has limited anatomical coverage. MRI is contraindicated for patients with pacemakers and claustrophobia.

There are some limitations to this study. First, the reviewers evaluated the images, were aware of the study's purposes and knew that IS was present in the patients. This may have resulted in bias. Second, aliasing artifacts can be seen if 3D-SPACE-VFAM exams are not performed with slabselective mode, as observed in our eight excluded cases. This situation may affect our results related to the flow-void phenomenon scores. Third, we have obtained the PC-MRI images through the lower thoracic region. The scoliotic apex of all patients was not in this region. CSF velocity values at
Fig. 3 3D-SPACE-VFAM images of a patient (MT group) with grade 3 flow-void phenomenon (arrows in A, B, $\mathbf{D}, \mathbf{E})$ and a control case with grade 1 flow-void phenomenon $(\mathbf{C}, \mathbf{F}, \mathbf{G})$

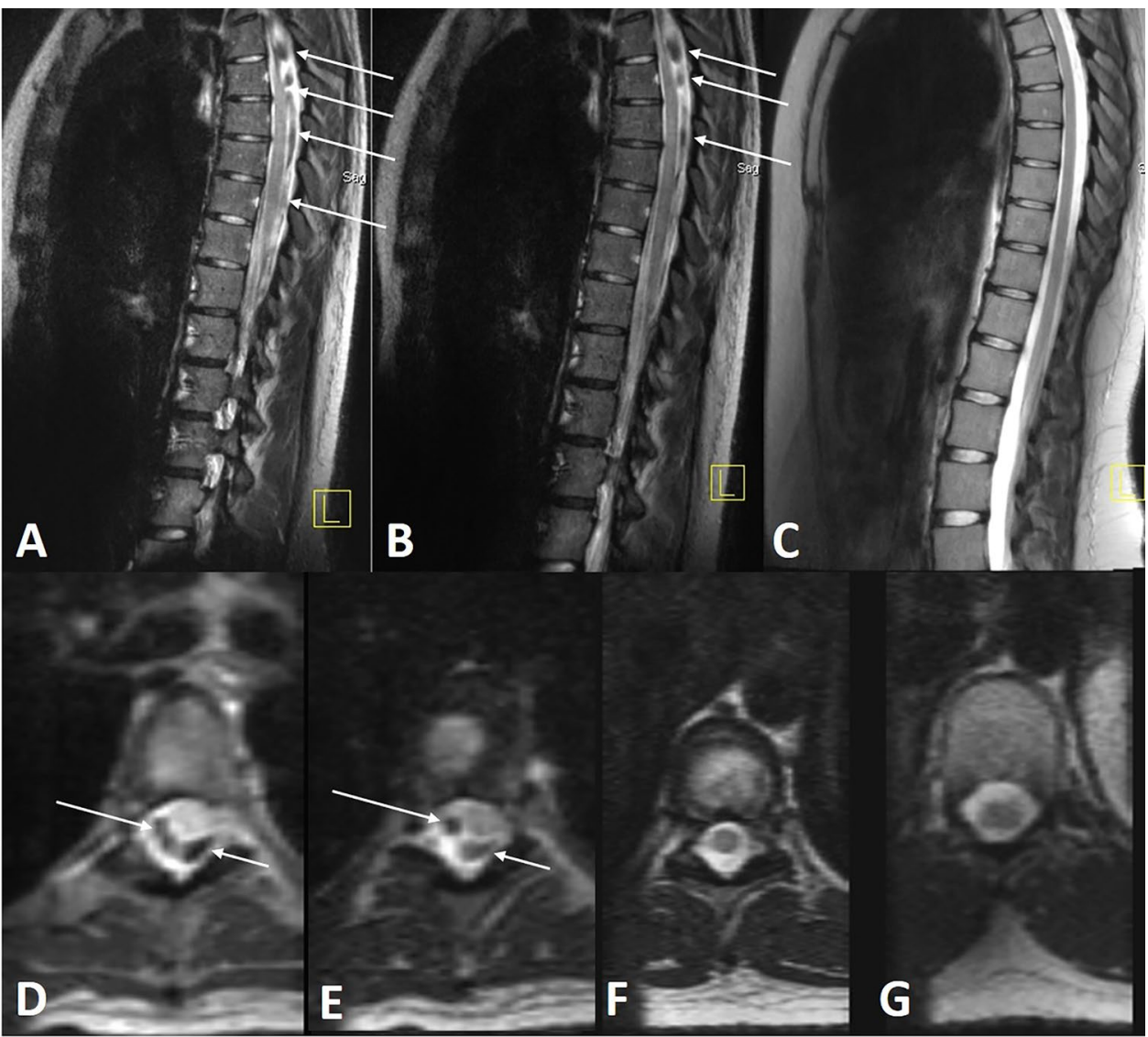


Table 5 Flow-void phenomenon scores of the groups on 3D-SPACE images

\begin{tabular}{|c|c|c|c|c|}
\hline & & \multicolumn{2}{|l|}{ Groups } & \multirow[t]{2}{*}{ Tota } \\
\hline & & Control & Patient & \\
\hline \multirow[t]{4}{*}{ Flow-void scores } & 1 & 2 & 2 & 4 \\
\hline & 2 & 3 & 7 & 11 \\
\hline & 3 & 1 & 17 & 22 \\
\hline & 4 & 0 & 8 & 12 \\
\hline Total & & 6 & 34 & 40 \\
\hline
\end{tabular}

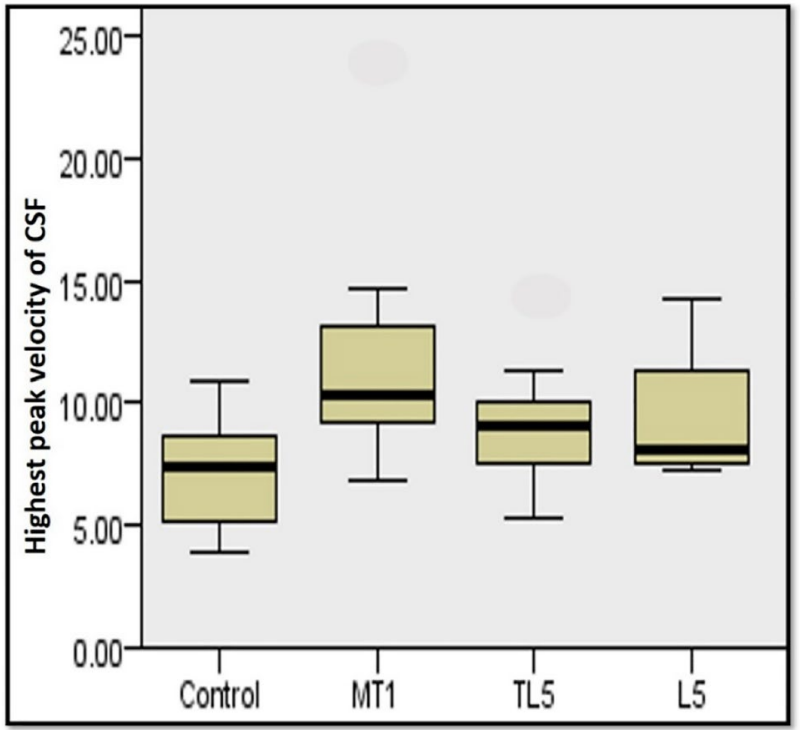

Fig. 4 The highest peak velocity of CSF values of the controls and patients. Notes =MT1: main thoracic, Lenke type 1, TL5: thoracolumbar Lenke type 5, L5: Lumbar, Lenke type 5 the apex may be observed higher. Customized and standardized measurements at different levels of the scoliotic curve are necessary for all patients. Fourth, our findings for both groups were obtained from a relatively small population. Finally, IS is frequently seen in adolescents, as mentioned above. However, all cases in our study were not adolescents, but most of them are adolescents. Carrying out specific studies for the adolescent age group can give more striking results.

\section{Future perspectives}

Characteristics and fluid dynamics of CSF flow in humans are still poorly understood [18]. Pulsatile movements and flow characteristics of CSF are dependent on the heartbeat and respiration features of each case [18]. According to a preliminary study, respiratory effects were more pronounced for abdominal than thoracic breathing, at thoracic-eight/ lumbar-three levels [19]. The abnormalities in spinal CSF flow or velocities observed in scoliosis patients may also be related to characteristic respiratory disturbances due to the thoracic cavity's morphological changes. In our study, we obtained PC-MRI data with only ECG gating. It is also necessary to investigate whether similar findings can be observed with respiratory gating.

All present treatment procedures may be altered as a result of the MRI findings that they are possible to predict the curves that will progress rapidly from the beginning or initial diagnosis of the disease. Monitoring CSF flow abnormalities by MRI can make the treatment of the patients with scoliosis more effective. These techniques may also help determine the efficacy of some physiotherapeutic methods like the Schroth technique or whether to use a brace or decide on early surgical intervention before curve progression. As a result, prospective, longitudinal, and/or more extensive/specific studies are needed to assess CSF flow abnormalities with IS.

Table 6 Intra-reader and inter-reader agreements of PC-MRI measurements and flow-void phenomenon scores

\begin{tabular}{lllll}
\hline Intra-reader agreement & ICC & $p$ Value & Inter-reader agreement & ICC \\
\hline Highest maximum spine movement & 0.950 & 0.000 & Highest maximum spine movement & 0.999 \\
Maximum spine movement & 0.937 & 0.000 & Maximum spine movement & 0.000 \\
Average spine movement & 0.961 & 0.000 & Average spine movement & 0.000 \\
Highest peak CSF velocity & 0.883 & 0.000 & Highest peak CSF velocity & 0.963 \\
Mean peak CSF velocity & 0.866 & 0.000 & Mean peak CSF velocity & 0.976 \\
Average CSF velocity & 0.989 & 0.000 & Average CSF velocity & 0.972 \\
Average over range & 0.954 & 0.000 & Average over range & 0.716 \\
Net forward volume & 0.952 & 0.000 & Net forward volume & 0.000 \\
Area & 0.824 & 0.000 & Area & 0.003 \\
Intra-reader agreement & Kappa & $\boldsymbol{p}$ Value & Inter-reader agreement & 0.000 \\
Flow-void phenomenon & 0.494 & 0.005 & Flow-void phenomenon & 0.000 \\
\hline
\end{tabular}




\section{Conclusion}

The evidence from the PC-MRI results revealed that hyperdynamic or altered CSF flow is present in patients with IS in parallel to the literature. 3D-SPACE-VFAM is a non-invasive, reliable, safe, and effective method for the evaluation of the spine. Our results can serve or prepare the ground for further research, such as predetermining and/or preventing the curve progression and investigating the effects of different conservative and surgical treatment protocols by analyzing the CSF flow. Therefore, there is a clinical impact from our findings.

Acknowledgements The authors thank neurosurgeon Ercan Bal, orthopedist Atıf Aksekili, pharmacist Selin Arslan, and linguist Guven Mengu for their contributions.

\section{Declarations}

Ethical approval All procedures performed in the studies involving human participants were in accordance with the ethical standards of the institutional and/or national research committee and with the 1964 Helsinki Declaration and its later amendments or comparable ethical standards.

Informed consent Informed consent was obtained from all individual participants included in the study.

Conflict of interest The authors declare that they have no conflict of interest.

\section{References}

1. Kim H, Kim HS, Moon ES et al (2010) Scoliosis imaging: what radiologists should know. Radiographics 30(7):1823-1842

2. Van Gennip JLM, Boswell CW, Ciruna B (2018) Neuroinflammatory signals drive spinal curve formation in zebrafish models of idiopathic scoliosis. Sci Adv 4(12):eaav1781

3. Faizah MZ, Ng KL, Te BC et al (2016) Association of Cobb angle progression and neuraxial abnormality on MRI in asymptomatic adolescent idiopathic scoliosis. Med J Malaysia 71(3):122-125

4. Grimes DT, Boswell CW, Morante NF, Henkelman RM, Burdine RD, Ciruna B (2016) Zebrafish models of idiopathic scoliosis link cerebrospinal fluid flow defects to spine curvature. Science 352(6291):1341-1344

5. Duchaussoy T, Lacoste M, Norberciak L, Decaudain J, Verclytte S, Budzik JF (2019) Preoperative assessment of idiopathic scoliosis in adolescent and young adult with three-dimensional T2-weighted spin-echo MRI. Diagn Interv Imaging 100(6):371-379
6. Zhao Z, Li T, Bi N et al (2020) Continuous hypodynamic change of cerebrospinal fluid flow as a potential factor working for experimental scoliotic formation. Sci Rep 10(1):6821

7. Sternberg JR, Prendergast AE, Brosse L et al (2018) Pkd211 is required for mechanoception in cerebrospinal fluid-contacting neurons and maintenance of spine curvature. Nat Commun 9(1):3804

8. Algin O, Turkbey B (2012) Evaluation of aqueductal stenosis by $3 \mathrm{D}$ sampling perfection with application-optimized contrasts using different flip-angle evolutions sequence: preliminary results with 3 T MR imaging. AJNR Am J Neuroradiol 33(4):740-746

9. Demirkiran G, Dede O, Yalcin N, Akel I, Marcucio R, Acaroglu E (2014) Selective estrogen receptor modulation prevents scoliotic curve progression: radiologic and histomorphometric study on a bipedal C57B16 mice model. Eur Spine J 23(2):455-462

10. Noureldine MHA, Shimony N, Jallo GI, Groves ML (2019) Scoliosis in patients with Chiari malformation type I. Childs Nerv Syst 35(10): 1853-1862

11. Oliazadeh N, Gorman KF, Eveleigh R, Bourque G, Moreau A (2017) Identification of elongated primary cilia with impaired mechanotransduction in idiopathic scoliosis patients. Sci Rep 7:44260

12. Boswell CW, Ciruna B (2017) Understanding idiopathic scoliosis: a new zebrafish school of thought. Trends Genet 33(3):183-196

13. Terhune EA, Baschal EE, Miller NH (2018) Genetics and functional pathology of idiopathic scoliosis. In: Kusumi K, Dunwoodie $\mathrm{S}$ (eds). The genetics and development of scoliosis. Springer, Cham https://doi.org/10.1007/978-3-319-90149-7_7

14. Takano S, Horie T, Niwa T et al (2019) Quantitative assessment of flow velocity of cerebrospinal fluid using 2D phase-contrast MRI in adolescent idiopathic scoliosis. ECR / C-0791. https://doi.org/ 10.26044/ecr2019/C-0791

15. Scaramuzzo L, Giudici F, Archetti M, Minoia L, Zagra A, Bongetta D (2019) Clinical relevance of preoperative MRI in adolescent idiopathic scoliosis: is hydromyelia a predictive factor of intraoperative electrophysiological monitoring alterations? Clin Spine Surg 32(4):E183-187

16. Tully PA, Edwards BA, Mograby O et al (2018) Should all pediatric patients with presumed idiopathic scoliosis undergo MRI screening for neuro-axial disease? Childs Nerv Syst 34(11):2173-2178

17. Hall FM, Kim S, Yoon CS, Chung TS, Kim H (2011) Re: more about scoliosis imaging for radiologists. Radiographics 31(4):1191; author reply 1191-2

18. Dreha-Kulaczewski S, Joseph AA, Merboldt KD, Ludwig HC, Gärtner J, Frahm J (2017) Identification of the upward movement of human CSF in vivo and its relation to the brain venous system. J Neurosci 37(9):2395-2402

19. Aktas G, Kollmeier JM, Joseph AA et al (2019) Spinal CSF flow in response to forced thoracic and abdominal respiration. Fluids Barriers CNS 16(1):10

Publisher's Note Springer Nature remains neutral with regard to jurisdictional claims in published maps and institutional affiliations. 